\title{
Dalla cardiochirurgia alla neurochirurgia: un caso di apoplessia ipofisaria dopo bypass aorto-coronarico con chiara evidenza RMN
}

\author{
Vittoria Ramunno ${ }^{1}$. Felicia Visconti ${ }^{1}$. Sabrina Chiloiro ${ }^{1}$ - Laura Rossi $^{1}$ - Antonella Giampietro ${ }^{1}$ - Alfredo Pontecorvi ${ }^{1}$. \\ Laura De Marinis ${ }^{1}$ · Antonio Bianchi ${ }^{1}$
}

Accettato: 27 maggio 2020 / Pubblicato online: 16 marzo 2021

(c) The Author(s) 2021

L'apoplessia ipofisaria $[1,2]$ è una sindrome conseguente a un infarto o un'emorragia all'interno di un adenoma ipofisario. Essa può verificarsi anche in seguito a intervento di cardiochirurgia [3, 4], in presenza di fattori precipitanti tra cui: anticoagulazione sistemica, ipotensione, ridotta ossigenazione tissutale, edema indotto dalla procedura, emodiluizione, ventilazione a pressione positiva.

Un uomo di 71 anni, con occasionale riscontro di ischemia cardiaca e coronarografia diagnostica per malattia coronarica trivasale, veniva sottoposto a intervento cardiochirurgico di doppio bypass aorto-coronarico. Nel postoperatorio insorgevano bradipsichismo e stato soporoso, con TC del cranio risultata negativa per eventi ischemici/emorragici acuti ma con evidenza di lesione espansiva sellare (Fig. 1). La valutazione endocrinologica includeva dosaggi degli ormoni ipofisari, indicativi di panipopituitarismo anteriore, non noto in anamnesi e trattato con terapia sostitutiva con idrocortisone e levotiroxina, con beneficio clinico. Alla ripresa dello stato di vigilanza riscontro di diplopia in tutte le posizioni dello sguardo, accompagnata da deficit del IV e del VI nervo cranico di destra. La RMN ipofisi (Fig. 2) confermava la lesione espansiva sellare/sovrasellare, in contatto con il chiasma ottico con invasione del seno cavernoso di destra, compatibile con macroadenoma, con marcato aspetto disomogeneo. Dopo adeguato periodo di sospensione della terapia antiaggregante e preparazione con idrocortisone endovenoso, il paziente

$凶$ V. Ramunno

vittoria.ramunno@gmail.com

1 U.O.S. di Patologia Ipofisaria, U.O.C. di Endocrinologia e Diabetologia, Fondazione Policlinico Universitario Agostino Gemelli IRCCS, Università Cattolica del Sacro Cuore, Roma, Italia

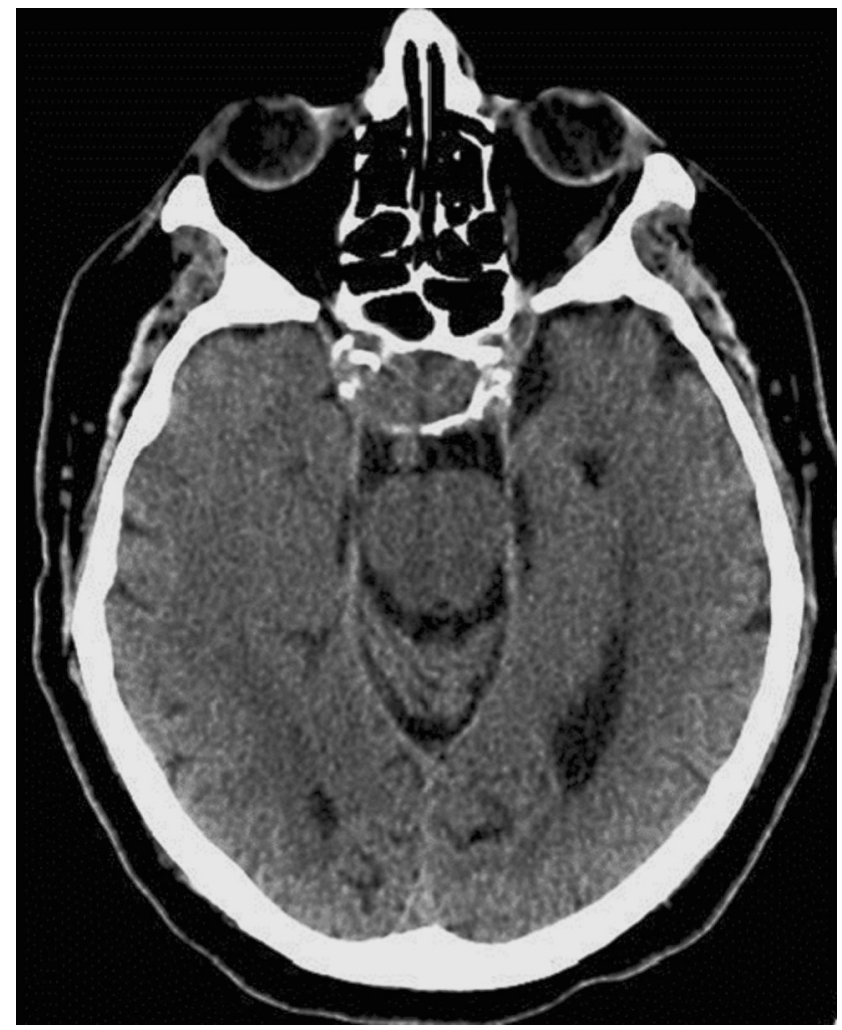

Fig. 1 TC encefalo: l'immagine mostra la lesione espansiva sellare

veniva sottoposto a intervento neurochirurgico di resezione di macroadenoma ipofisario per via trans-sfenoidale. Durante l'intervento riscontro di importante componente necrotico-colliquativa, confermata all'esame istologico definitivo, conclusivo per adenoma ipofisario apoplettico. A due mesi dall'intervento persistenza di lieve diplopia e buon compenso endocrino in corso di terapia ormonale sostitutiva. 


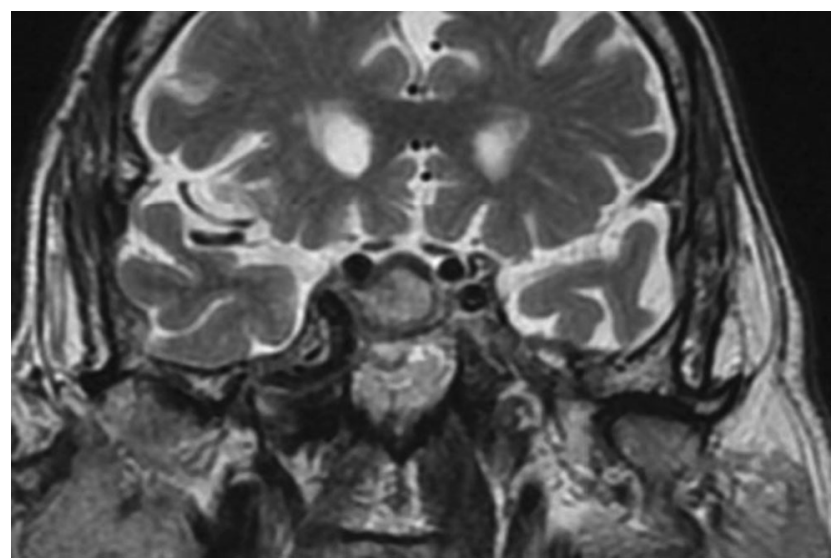

Fig. 2 RM ipofisi dinamica: l'immagine mostra una lesione espansiva disomogenea sellare/sovrasellare, in contatto con il chiasma ottico con invasione del seno cavernoso di destra

Funding Note Open access funding provided by Università Cattolica del Sacro Cuore within the CRUI-CARE Agreement.

Conflitto di interesse Gli autori Vittoria Ramunno, Felicia Visconti, Sabrina Chiloiro, Laura Rossi, Antonella Giampietro, Alfredo Pontecorvi, Laura De Marinis e Antonio Bianchi dichiarano di non avere conflitti di interesse.

Consenso informato Per lo studio presentato in questo articolo è stato ottenuto il consenso informato al paziente.

Studi sugli animali Gli autori di questo articolo non hanno eseguito studi sugli animali.
Nota della casa editrice Springer Nature rimane neutrale in riguardo alle rivendicazioni giurisdizionali nelle mappe pubblicate e nelle affiliazioni istituzionali.

Open Access This article is licensed under a Creative Commons Attribution 4.0 International License, which permits use, sharing, adaptation, distribution and reproduction in any medium or format, as long as you give appropriate credit to the original author(s) and the source, provide a link to the Creative Commons licence, and indicate if changes were made. The images or other third party material in this article are included in the article's Creative Commons licence, unless indicated otherwise in a credit line to the material. If material is not included in the article's Creative Commons licence and your intended use is not permitted by statutory regulation or exceeds the permitted use, you will need to obtain permission directly from the copyright holder. To view a copy of this licence, visit http://creativecommons.org/licenses/by/4.0/.

\section{Bibliografia}

1. Cannavò S, Albani A, Granata F et al (2014) L'apoplessia ipofisaria: riconoscerla rapidamente e trattarla correttamente. Endocrinologo 15:165-171

2. Messina E, Ferraù F, Cannavò S (2019) Gestione clinica dell'apoplessia ipofisaria. Endocrinologo 20:341-345

3. Hidiroglu M, Kucuker A, Ucaroglu E, Kucuker SA (2010) Pituitary apoplexy after cardiac surgery. Ann Thorac Surg 89(5):1635-1637

4. Mattke AF, Vender JR, Anstadt MR (2002) Pituitary apoplexy presenting as Addisonian crisis after coronary artery bypass grafting. Tex Heart Inst J 29:193-199 